\title{
Microelectromechanical Switches for Phased Array Antennas
}

\author{
George E. Ponchak, Rainee N. Simons, Maximillian Scardelletti, \\ and Nicholas C. Varaljay \\ NASA Glenn Research Center \\ 21000 Brookpark Rd, Cleveland, OH 44135
}

\begin{abstract}
Preliminary results are presented on the fabrication and testing of a MicroElectro-Mechanical (MEM) microstrip series switch. This switch is being developed for use in a K-band phased array antenna that NASA will use for communication links in its Earth orbiting satellites. Preliminary insertion loss and isolation measurements are presented.
\end{abstract}

\section{Introduction}

NASA, military, and commercial satellites use phased array antennas on their Geostationary Earth Orbiting (GEO) satellites for beam shaping and steering [1]. By generating several spot beams to cover the conus and switching them in turn to transmit/receive a RF signal to or from a region, the frequency spectrum is more efficiently used. For the new constellations of Medium and Low Earth Orbiting (MEO and LEO) satellite systems, phased array antennas are required to track the satellite as it rapidly moves across the sky.

Typically, electronically steered phased array antenna systems are made of large numbers of radiating elements that each have their own phase shifter, gain control amplifier, and power amplifier immediately behind the radiating element. In addition, some systems include a local oscillator and a mixer behind each element while other systems perform the mixing operation only once. In either case, the modulated RF signal is fed to each element through a corporate feed network. This is shown schematically in Figure 1. By changing the phase and magnitude of the RF signal at each antenna element in a controlled way, the radiated beam may be steered and shaped.

Several issues have prevented the more wide spread use of phased array antennas. First, they are expensive because they require a full transmit/receive MMIC module behind each antenna element, and typical phased array antennas require more than several thousand antenna elements. Second, designing the RF feed network and packaging the system is difficult at higher frequencies because the antenna element spacing scales linearly with frequency but the RF circuits and transmission lines that comprise the antennas system do not. Third, MMIC power amplifiers are not very efficient. Therefore, substantial heat is generated that must be distributed, but because the power amplifiers are tightly packed immediately behind the antenna elements, thermal distribution is difficult. 


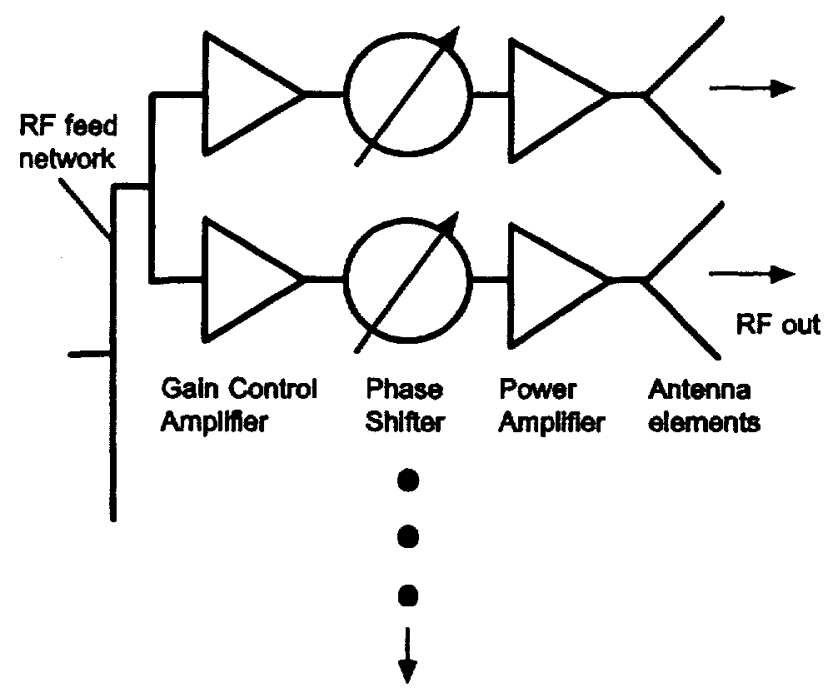

Figure 1: Schematic of phased array antenna system as it is currently implemented.

A partial solution to these problems is to use a single power amplifier for multiple antenna elements as shown in Figure 2. This reduces the number of components, the thermal distribution problems, and the cost. However, this is only possible if the insertion loss in the RF feed network and the phase shifter is small so the antenna efficiency does not suffer. With state of the art GaAs MESFET switches, five bit phase shifters typically have an insertion loss of $5 \mathrm{~dB}$ at $\mathrm{K}$ band [2]. This loss is too high to implement the antenna configuration shown in Figure 2.

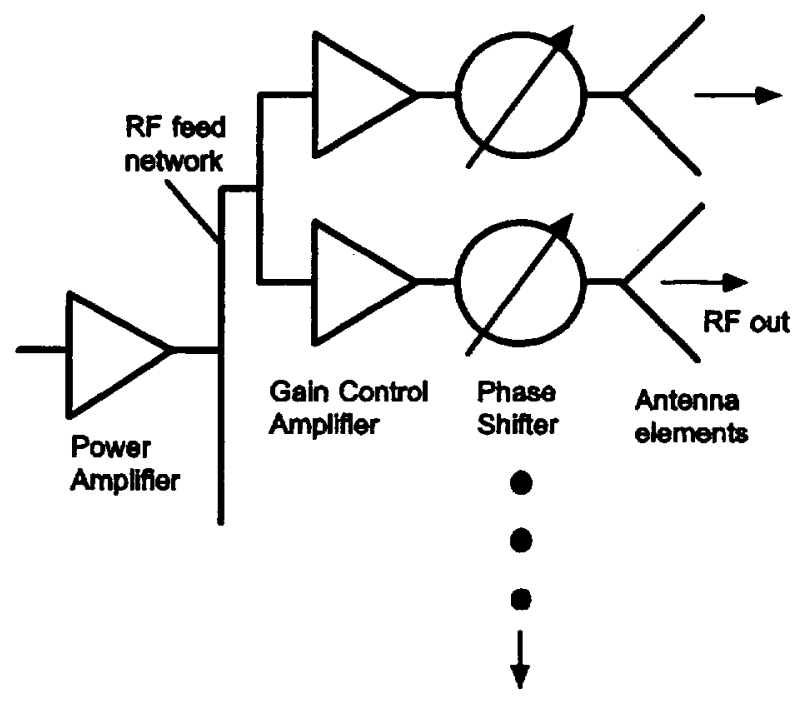

Figure 2: Schematic of phased array antenna system that is possible if losses in the $R F$ feed network and phase shifter are reduced.

MEMS switches have recently been demonstrated with an insertion loss of $0.1 \mathrm{~dB}$ [3], which should enable five bit phase shifters with less than $2 \mathrm{~dB}$ of insertion loss. In this paper, the preliminary results of a NASA GRC developed MEMS microstrip series switch is presented. 


\section{Switch Fabrication and Testing}

The microstrip series MEMS switch shown in Figure 3 is fabricated on a silicon wafer with a resistivity of $2500 \Omega$-cm. First, the microstrip lines are defined on the Si wafer using a liftoff process. The metal lines consist of $200 \AA \mathrm{Cr}$ and 1.5 $\mu \mathrm{m}$ of $\mathrm{Au}$. Normally, a $3000 \AA$ layer of $\mathrm{Si}_{3} \mathrm{~N}_{4}$ would be grown where the switch makes contact, but the $\mathrm{Si}_{3} \mathrm{~N}_{4}$ was not grown for this preliminary switch. Next, 2.1 $\mu \mathrm{m}$ of $\mathrm{SiO}_{2}$ is RF sputtered over the wafer to serve as the sacrificial layer. A hole is wet etched in the $\mathrm{SiO}_{2}$ for the cantilever anchor, and the cantilever is defined by liftoff technology. The cantilever consists of electron beam evaporated $200 \AA \mathrm{Cr}$ and $1.9 \mu \mathrm{m}$ of $\mathrm{Au}$. The cantilever is $225 \mu \mathrm{m}$ long and $150 \mu \mathrm{m}$ wide. Finally, the sacrificial layer is etched in $\mathrm{HF}$ and the cantilevers released using a $\mathrm{CO}_{2}$ super critical release. An array of holes in the cantilever assists in the removal of the sacrificial $\mathrm{SiO}_{2}$ layer. No ground plane is grown on the backside of the $\mathrm{Si}$ wafer.

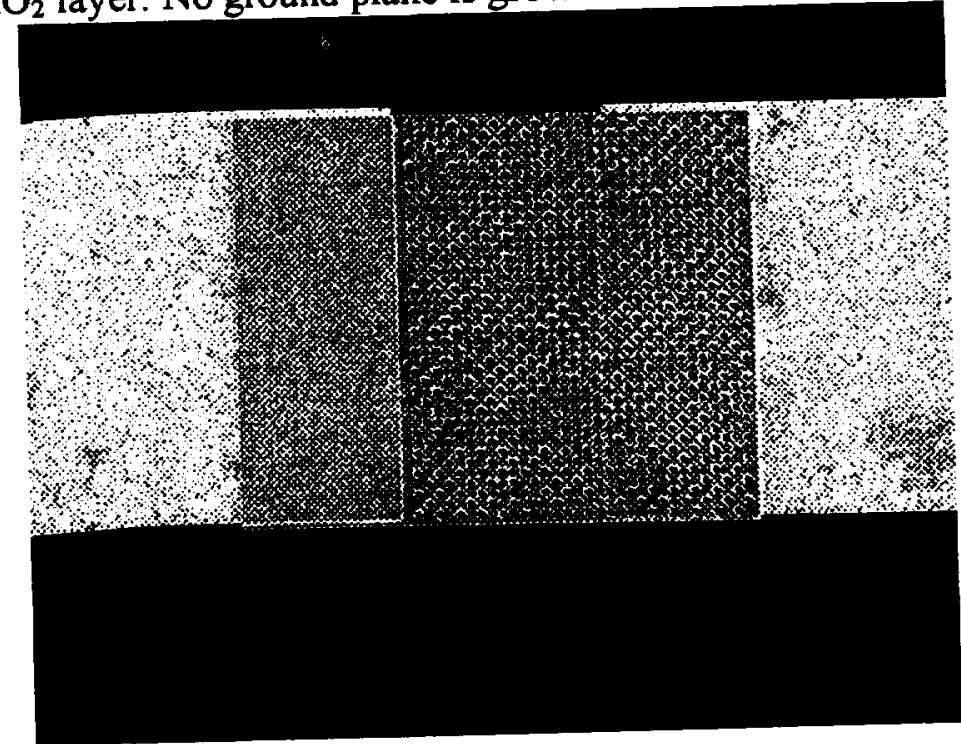

Figure 3: SEM micrograph of MEMS microstrip series switch (cantilever is $225 \mu \mathrm{m}$ long and $150 \mu \mathrm{m}$ wide).

To characterize the switch, the circuit is diced from the Si wafer and mounted on a Au coated Alumina carrier, which serves as the ground plane for the microstrip MEMS switch. Microstrip feed lines are fabricated on $245 \mu \mathrm{m}$ thick RT/Duroid 5880 substrates and mounted on the same Alumina carrier. Twenty-five micron thick $\mathrm{Au}$ wire bonds are used to connect the microstrip feed lines to the MEMS switch. On the Duroid substrate, Thru/Reflect/Line (TRL) calibration standards are defined and used for calibrating the measurement system to the edge of the microstrip on the Duroid. The switch is characterized on a vector network analyzer using RF probes. Bias to the switch is supplied through the RF probes using specially designed bias tees.

\section{Results}

The RF characteristics of this preliminary MEMS switch over the frequency range of 10 to $20 \mathrm{GHz}$ is shown in Figure 4 . Over most of the frequency band, the switch has $20 \mathrm{~dB}$ of isolation; however, if the switch had the expected insertion 
loss of less than $0.5 \mathrm{~dB}$, the isolation would have been greater than $30 \mathrm{~dB}$. Several factors contributed to the high insertion loss. First, the ground plane is not in intimate contact with the $\mathrm{Si}$ substrate. Second, the long bond wires introduced substantial inductance. A measured poor return loss confirms these observations. However, the switch fabrication also needs to be improved. The cantilever beam is bowed upward due to residual stress in the Au. In addition, the RF sputtered sacrificial layer is highly conformal. Therefore, the cantilever that is defined on top of the $\mathrm{SiO}_{2}$ is not flat, which tends to stiffen the beam. This resulted in the need for a high actuation voltage of $120 \mathrm{~V}$ to puli the cantilever beam down. Finally, residue under the cantilever prevented the beam from completely collapsing, which increased the insertion loss.

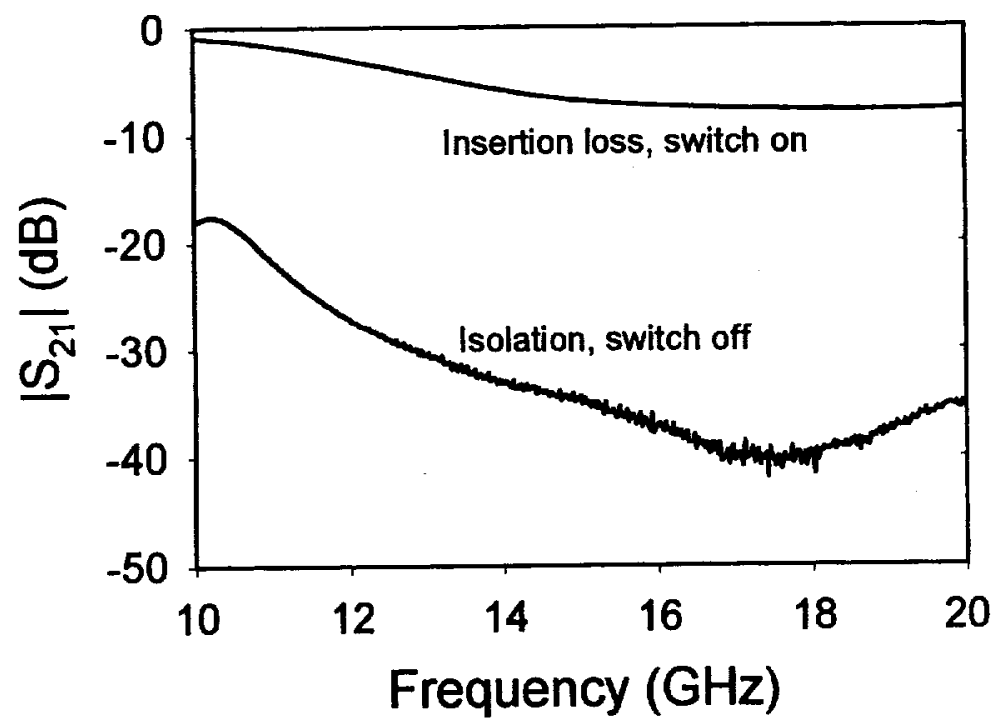

Figure 4: Measured insertion loss and isolation of microstrip MEMS switch.

\section{Conclusion}

Preliminary results of a MEMS microstrip series switch are presented. Although further improvements in the fabrication process are required, MEMS switches show great promise for the realization of new phased array system architectures.

\section{References}

1. J. V. Evans, "Proposed U.S. Multimedia Satellites," in IEEE Antennas and Propagation Society International Symposium 1998 Digest, Atlanta, GA, June 21-26, 1998, pp. 2-5.

2. D. A. Blackwell, D. E. Dawson, and D. C. Buck, "X-band MMIC switch with $70 \mathrm{~dB}$ isolation and $0.5 \mathrm{~dB}$ insertion loss," in IEEE 1995 Microwave and Millimeter-Wave Monolithic Integrated Circuits Symposium Digest, Orlando, FL, May 15-16, 1995, pp. 97-100.

3. S. P. Pacheco and L. P. B. Katehi, "Microelectromechanical K-band switching circuits," in 29th European Microwave Conference Digest, Munich, Germany, Oct. 5-7, 1999, pp. 45-48. 